\title{
Preface: Imaging and Measurements in Flow and Transport Phenomena
}

The focus of the journal continues to be on principles and applications of flow visualization techniques and related image processing algorithms for the quantification of the data recorded. Both aspects, data generation from experiments followed by data analysis and interpretation are important and the journal actively seeks articles in these directions. As a first step, it was felt that data generation could be given importance along with the information emerging from imaging in flow and heat transfer experiments. Accordingly, a special issue of the Journal of Flow Visualization and Image Processing entitled "Imaging and Measurements in Flow and Transport Phenomena" was conceived with the following announcement:

"Optical methods are nonintrusive, inertia free, and can image cross sections of the experimental apparatus. With the experiment suitably carried out, three dimensional physical domains with unsteady processes can be accommodated. The special issue is intended to provide the readers a glimpse of this exciting development in applications related to transport phenomena."

Imaging velocity and temperature fields over a cross section and as a function of time is required in several applications. If the region of interest is transparent, beam bending effects can be used to extract information about temperature. Similar principles can be used to determine concentration of salts dissolved in liquids. If the fluid is seeded with particles, velocity information can be extracted. Recent developments in imaging involve detection and tracking of interfaces and estimation of local, time-dependent wall stresses and fluxes. The fundamentals involved in such measurements

- both refractive index and scattering based, experimental setup, data analyses and retrieval, and specialized applications constitute the scope of the special issue.

The call was open for all authors. The editors also approached a few experts for their contributions. All articles were peer reviewed at length over a period of around four months.

The special issue is a collection of fourteen articles that covers a considerable spread of topics. Interferometry, schlieren, and shadowgraph are included, as are IR imaging and direct imaging of interfacial phenomena. Images arising from numerical simulation have been included where it was felt that equivalent experimental images would be difficult to record.

The editors of the special issue are thankful to the authors as well as to the reviewers of the submitted manuscripts for their contributions. They are also confident that the selection of articles will considerably enthuse researchers and practitioners of the subject.

Atul Srivastava, IIT Bombay (India)

K. Muralidhar, IIT Kanpur (India)

December 2018 